\title{
OPTIMISMO Y PERCEPCIÓN DE LA ACTIVIDAD FÍSICA EN USUARIOS DE UNA PLAZA URBANA DE MONTEVIDEO
}

\section{OPTIMISM AND PHYSICAL ACTIVITY PERCEPTION IN URBAN PARK USERS OF MONTEVIDEO}

\author{
Verónica Tutte Vallarino \\ Universidad Católica del Uruguay, Uruguay \\ Cecilia del Campo Vega \\ Instituto Universitario Asociación Cristiana de Jóvenes, Uruguay
}

\begin{abstract}
Resumen: Este trabajo se centra en el estudio de los perfiles optimista-pesimista en ejercitantes de una plaza urbana comunitaria, así como la percepción de actividad que tienen de sí mismos. Dentro de los objetivos planteados en esta investigación, está presente el interés profundizar y comparar las diferencias que se pudieran presentar entre estas variables y el género.

Se administró el Life Orientation Test (LOT-R) elaborado por Scheier y Carver (1985), en su versión española de Otero-López, Luengo, Romero, Triñanes, Gómez y Castro (1998) para valorar el perfil optimista-pesimista, mientras que para evaluar la percepción de actividad se realizó una pregunta, con un tipo de respuesta dicotómica, a 45 usuarios de edades comprendidas entre los 17 y 53 años.

En relación a los resultados encontrados, se pudo observar una diferencia estadísticamente significativa entre los factores optimismo-pesimismo, siendo el perfil optimista, el que presenta un amplio predominio, representando un 82 \% de la muestra total. No encontramos aquí, diferencias estadísticamente significativas entre el género y los factores. Asimismo, la variable percepción de actividad, sí presenta diferencias estadísticamente significativas en relación al género, cuyo resultado evidencia un amplio margen en favor de los hombres.

Por último, los resultados alcanzados nos permiten establecer que hay un predominio de personas optimistas que se perciben como activas, sin existir diferencias estadísticamente significativas entre los factores disposicionales: optimismo-pesimismo y la percepción de actividad: activo-pasivo.
\end{abstract}

Palabras clave: optimismo, percepción de actividad, usuario de plaza.

\begin{abstract}
This paper focuses on the study of the pessimistic-optimistic profiles in users of a community park, and the perception of their own physical activity.

One of our major interests is to compare and further the understanding of these variables, as well as to analyze how they relate to gender.

Pessimistic-optimistic profiles were evaluated using the Life Orientation Test (LOT-R) developed by Scheier and Carver (1985), and its Spanish version by Otero-López, Luengo, Romero, Triñanes, Gómez and Castro (1998), while perception of physical activity was evaluated by a single question with a dichotomous response. 45 park users, between 17 and 53 years old, took part in this study.

The results show a statistically significant bias in the optimism-pessimism factor; with the optimistic profile representing $82 \%$ of the sample. We did not find any statistically significant gender related bias in the optimism- pessimism factor. We have found a statistically significant gender related bias in the perception of own physical activity in relation to gender, with men showing higher scores.

Finally, the result allows us to establish that there is a predominance of optimistic people who see themselves as active persons. However no statistically significant differences were observed when optimism and active self perception, or pessimism and passive self perception, were analyzed.
\end{abstract}

Keywords: optimism, physical activity perceptions, park users.

Correspondencia: Verónica Tutte. Departamento de Ciencias Cognitivas y de la Salud. Facultad de Psicología. Universidad Católica del Uruguay. Correo Electrónico: vtutte@ucu.edu.uy. 


\section{INTRODUCCIÓN}

La depresión ha sido una presencia recurrente de estos últimos tiempos y el papel jugado por el pesimismo en la depresión (Beck, 1967) ha sido un factor determinante muy negativo. En contraposición a esto surge el interés por ahondar en nuevas temáticas y líneas de investigación que procuren el bienestar psicológico y que apunten a la prevención de patologías y al afrontamiento de situaciones adversas.

En los últimos años, las investigaciones sobre optimismo han abarcado diferentes contextos entre los que se encuentran el educativo y la actividad física. Ambos contextos comparten de hecho la necesidad de afrontar retos y superar adversidades, y sobre todo la necesidad de superar evaluaciones, numéricas y en ocasiones sociales (Ortín, Ortega, López, y Olmedilla, 2011).

La revisión de la literatura en Psicología (Romero, García-Mas \& Brustadl, 2009), indica que el optimismo parece jugar un importante papel en el uso de conductas de afrontamiento adaptativas, así como en el bienestar psicológico y físico y parece ser un importante predictor de la enfermedad, tanto psicológica como física.

En el ámbito del deporte y el ejercicio físico, el optimismo parece tener relación con variables que determinan de forma directa o indirecta el rendimiento deportivo y la participación en programas de actividad física.

El optimismo es un constructo de la personalidad que actúa como factor determinante cuando el deportista se encuentra en situaciones de presión (Seligman, 2004), marcando en numerosas ocasiones la diferencia entre como los deportistas gestionan las situaciones adversas y afrontan las dificultades en situaciones similares (Martin-Krumm, Sarrazin, Peterson y Famose, 2003; Seligman, Nolen-Hoeksema, Thornton y Thornton,1990).

De las diferentes aproximaciones al concepto de optimismo, encontramos dos, que nos permiten adentrarnos a este término. El primer enfoque, se basa en los trabajos de Scheier y Carver (1985) que toman como punto de partida un modelo de autorregulación de conducta. Este modelo parte de la idea de que cuando surgen dificultades, las expectativas favorables incrementan los esfuerzos de las personas para alcanzar objetivos, en tanto que las expectativas desfavorables reducen tales esfuerzos, a veces hasta el punto de desentenderse totalmente de la tarea. Dentro de este modelo, el optimismo y el pesimismo serían considerados como expectativas generalizadas (favorables y desfavorables respectivamente) acerca de las cosas que le suceden a uno en la vida. Tales expectativas se consideran además como disposiciones estables y de allí la concepción que estos autores plantean de optimismo disposicional (Scheier et al., 1985). Ha sido Seligman (2000) quien ha dado inicio a un nuevo campo de la psicología, denominado "Psicología Positiva", en esta nueva área se comienzan a aglutinar e integrar todos aquellos conceptos relativos al bienestar, la felicidad y los enfoques más positivos y optimistas de la salud mental dejando el foco más tradicionalista en la enfermedad o los estados negativos de salud mentaL (Romero, et al., 2009).

Por otro lado, el optimismo se ha estudiado desde la teoría de las pautas explicativas, (Abramson, Seligman y Teasdale, 1978), y que tiene su origen en las teorías de las atribuciones más clásicas (Weiner et al., 1971). Los estilos explicativos se refieren a la forma que las personas dan explicación a los sucesos que le ocurren (Isaacowitz, 2005; Shapcott, Bloom, Johnston, Loughead y Delaney, 2007).

En relación a la otra variable de nuestro estudio, la percepción de actividad, no hay una definición específica del término, por ello nos basaremos en la psicología del control para poder comprender su significado, alcance e importancia. La Psicología del Control es entendida como el conjunto de implicaciones derivadas del control conductual, es decir, de la existencia de contingencias entre la conducta del sujeto o de otros individuos y la obtención de determinados resultados (Fernández, Álvarez, Blasco, Doval, Sanz, 1998). Por tanto, se podría decir que la percepción de control se asocia a saber lo que se quiere, ser capaz de identificar los modos para conseguirlo, cambiar los objetivos cuando los iniciales no son factibles, ser capaz de reconocer las propias habilidades, saber incrementarlas si son necesarias y decidir cuándo merece la pena ejercer control y cuándo no (Bárez, 2002). En este caso, la conducta a controlar es la de, realizar o no, ejercicio físico de forma regular. 
Consideramos importante hacer referencia también a uno de sus componentes: la autoeficacia, que indica que el sujeto valora que el agente (él mismo), es capaz de desarrollar unos medios, que se presupone que permiten conseguir unos determinados resultados deseados por el individuo. La autoeficacia es una valoración de capacidades de ejecución, es decir una predicción del éxito conductual, (Bandura, 1982). De allí que cuando indagamos por la percepción de actividad, de alguna forma el individuo se cuestiona las habilidades con las que cuenta para poder realizar ejercicio físico.

La consecuencia de este razonamiento determinará lo que realmente haga el sujeto ante una situación en concreto y la reacción emocional que expresará. En general se relaciona la percepción de control de la actividad con la manera de afrontar (Carver et al., 2000). Así pues, se mantiene la hipótesis de que en situaciones percibidas como controlables, como lo es el poder realizar ejercicio físico, se pondrán en marcha estrategias de afrontamiento activas, que se centrarán en la solución de la misma.

Al referirnos al concepto de ejercicio físico, entendemos al mismo como toda actividad física que se realiza de "forma planificada, ordenada, repetida y deliberada, dirigida a la mejora de la condición física" (Caspersen, Powell y Christenson, 1985, p. 126). Los individuos que realicen esta actividad de forma regular, podrán encontrar en esta conducta un hábito saludable, que podría actuar como un factor protector de la salud integral y por ende del bienestar psicológico.

La percepción de control de la actividad y el optimismo disposicional: optimismo-pesimismo, podrían direccionarse de forma similar, pues ambos modelos conciben que cuando hay percepción de control y hay optimismo, los individuos ponen en funcionamiento estrategias de afrontamiento más adaptativas, que les permitirán llevar adelante la conducta que el individuo debe desarrollar.

De ahí que los objetivos planteados para este trabajo de investigación, presenten el interés de profundizar y comparar las diferencias que se pudieran presentar entre estas variables y el género.

\section{MÉTODO}

\subsection{Participantes}

Este estudio cuenta con 45 personas que realizan ejercicio en una plaza comunitaria en el centro de la ciudad de Montevideo, Uruguay. Las mediciones se realizaron entrando en la primavera, en la Plaza Liber Seregni, considerada la primera plaza urbana del Uruguay. Las edades de los participantes se encuentran entre los 17 y 53 años con una media de edad de 23.5 ( $M=$ 23.49; $D S=7.71$ ).

\subsection{Instrumentos}

El constructo optimismo fue evaluado con el cuestionario LOT-R, en su versión española de Otero-López, Luengo, Romero, Triñanes, Gómez y Castro (1998). El LOT-R es una revisión del Life Orientation Test (Scheier y Carver, 1985), llevada a cabo por Scheier, Carver y Bridges, (1994). Ferrando, Chico y Tous (2002), realizan un análisis psicométrico de la prueba revisada, obteniendo propiedades muy similares a las de la versión original. Está compuesto por 10 ítems, en los que los sujetos indican el grado de acuerdo o desacuerdo, con afirmaciones como "en tiempos difíciles, generalmente espero lo mejor", usando una escala de 5 puntos, donde 0 (muy en desacuerdo) y 4 (muy de acuerdo). De los 10 ítems, 3 se muestran en sentido positivo y 3 en sentido negativo. Los 4 ítems restantes son de control o relleno. Respecto a la corrección e interpretación de la prueba, aparecen dos opciones (Ferrando et al. 2002). Por un lado la medición de cada disposición por separado y por otro la medición del optimismo total al revertir los ítems redactados en sentido negativo. Los diferentes trabajos factoriales apoyan la obtención de los dos factores (Mroczek, Spiro, Aldwin, Ozer y Bosse, 1993; Myers y Steed, 1999). En este trabajo, con el objetivo de conocer la tendencia optimista-pesimista, a los valores obtenidos por el participante en el optimismo se le restó los obtenidos por el pesimismo. Se consideró que la tendencia de un participante era optimista si obtenía valores positivos, y pesimista, si obtenía valores negativos. En relación a la fiabilidad de los dos factores, en estudios recientes (Ortín, Garcés de los Fayos, Gosalvez, Ortega y OImedilla, 2011) han obtenido una valor de alfa de Cronbach de .711 para la subescala optimismo y .706 para la subescala de pesimismo. 
Para evaluar la percepción de actividad se realizaron dos preguntas. La primera indagaba la frecuencia de concurrencia a la plaza para hacer ejercicio físico “¿Cada cuánto viene a la plaza?”, de tipo abierta aceptando respuestas que luego se categorizaron; y la otra hace referencia a la percepción de actividad "¿Soy físicamente activo?", siendo dos las posibilidades de respuestas: 1- sí; y 2-no.

\subsection{Procedimiento}

Esta investigación se desarrolló en el transcurso de dos meses del presente año.

Los ejercitantes decidieron colaborar firmando un consentimiento informado, en donde aceptaban participar de esta investigación y se les explicaba los objetivos y la duración de la misma. Se administró el cuestionario y las preguntas de forma individual, con una duración aproximada de 10 minutos.

Una vez obtenida la información a partir de las técnicas empleadas es que procedimos a la corrección y al análisis de los datos.

\subsection{Análisis de Resultados}

Para el análisis de las subescalas de pesimismo y optimismo del cuestionario LOT-R, se han realizado diversos análisis, de acuerdo con las posibles maneras en que este cuestionario puede ser interpretado. Cuando analizamos por separado cada subescala estamos frente a variables continuas que pueden obtener valores del 1 al 12. En este caso, para corroborar la posible existencia de diferencias estadísticas significativas entre los sujetos optimistas y pesimistas, se empleó la prueba paramétrica (pues el análisis de asimetría nos determinaba que estamos frente a una variable que presenta una distribución normal) T para muestras relacionadas. A su vez, cuando analizamos los factores del LOT-R, desde el perfil disposicional, que interpreta un único resultado a partir de la diferencia de los valores obtenidos de la subescala optimismo, menos los valores de la subescala pesimismo, nos encontramos con una variable categórica que cuenta con dos posibilidades de respuesta: perfil optimista (valores positivos de entre 0 a 12), o un perfil pesimista (valores negativos de entre -12 a 0 ). Para el análisis de este perfil y de todas las variables categorizadas se utilizó la prueba de Chi-cuadrado.
Todos los análisis estadísticos se han realizado con un nivel de significación de $p \leq .05$ (Ntoumanis, 2001), a través del paquete estadístico SPSS 18.0

\section{RESULTADOS}

A continuación presentaremos el análisis descriptivo de cada una de las variables: Perfil optimista-pesimista y la percepción de autoeficacia, tanto en el total de la muestra $(N=45)$, y también de acuerdo al género.

En la Tabla 1, expondremos la frecuencia y porcentajes de los perfiles optimistas vs los pesimistas en la muestra total y por género.

Tabla 1.

Frecuencias y porcentajes de los perfiles optimista-pesimista

\begin{tabular}{|l|l|l|r|}
\hline \multicolumn{1}{|c|}{ Variable } & \multicolumn{1}{c|}{ Mujer } & \multicolumn{1}{c|}{ Hombre } & Total \\
\hline Optimista & $19(86.4 \%)$ & $5(21.7 \%)$ & $37(82.2 \%)$ \\
\hline Pesimista & $3(13.6 \%)$ & $18(78.3 \%)$ & $8(17.8 \%)$ \\
\hline
\end{tabular}

Los resultados encontrados, evidencian que tanto en el total de la muestra, como en los hombres y mujeres existe un amplio predominio del perfil optimista, representando un $82 \%$ de la muestra total. A partir del análisis de Chi-cuadrado, observamos que no existen diferencias estadísticamente significativas entre el género y estos dos perfiles disposicionales $\left(X^{2}=, 505^{\mathrm{a}} ; p=, 477\right)$.

En lo que respecta a los dos factores analizados por el Life Orientation Test, que hacen referencia al optimismo y pesimismo y que se interpretan de forma independiente, nos propusimos analizar si existen diferencias estadísticas entre ambos. Al tratarse de una variable continua (se evalúa en una puntuación directa de 1 a 12 , permitiendo jerarquizar entre los resultados) y que presenta una distribución normal en este caso, es que procedimos a realizar la prueba $T$ para muestras relacionadas.

A partir de los resultados observados en la Tabla 2, podemos establecer que existen diferencias estadísticamente significativas entre los factores optimismo y pesimismo de nuestra muestra $(p=.000)$. 
Tabla 2.

Prueba T para muestras relacionadas

\begin{tabular}{|l|c|c|c|c|c|c|}
\hline & Media & $\begin{array}{c}\text { Desviación } \\
\text { Tipica }\end{array}$ & $\begin{array}{c}\text { Error tip. de } \\
\text { la media }\end{array}$ & t & gl & Sig. \\
\hline $\begin{array}{l}\text { Optimista- } \\
\text { Pesimista }\end{array}$ & 4,68 & 3,78 &, 56392 & 8,3 & 44 &, 000 \\
\hline
\end{tabular}

En lo que refiere a la variable percepción de actividad, en la Tabla 3, presentaremos los datos relacionados al porcentaje y frecuencia de personas que se consideran activas vs pasivas, tanto en el total de la muestra, así como por género.

Tabla 3.

Frecuencias y porcentajes de la percepción de actividad

\begin{tabular}{|l|l|l|l|}
\hline \multicolumn{1}{|c|}{ Variable } & \multicolumn{1}{c|}{ Mujer } & \multicolumn{1}{c|}{ Hombre } & \multicolumn{1}{c|}{ Total } \\
\hline Activo & $13(59.1 \%)$ & $21(91.3 \%)$ & $34(75.6)$ \\
\hline Pasivo & $9(40.9 \%)$ & $2(8.7 \%)$ & $11(24.4 \%)$ \\
\hline
\end{tabular}

De los datos expuestos en la Tabla 3, podemos observar que los porcentajes varían de acuerdo al género, existiendo una clara predominancia en los hombres de percibirse como potencialmente activos (92\%), frente a una percepción más moderada en las mujeres, siendo el $59 \%$ de las mismas las que se perciben como activas.

Frente a esta amplia diferencia en la percepción de actividad por parte de hombres y mujeres, es que realizamos el análisis de Chicuadrado para analizar la posible existencia de diferencias estadísticamente significativas entre el género y la percepción de actividad.

A continuación presentamos la Tabla 4.

Tabla 4.

Estadístico de Chi-cuadrado para la variable percepción de actividad y género

\begin{tabular}{|c|c|c|c|}
\hline & Valor & gl & Sig.Asintótica (Bilateral) \\
\hline $\begin{array}{c}\text { Chi-cuadrado de } \\
\text { Pearson }\end{array}$ & $6,318^{\mathrm{a}}$ & 1 &, 012 \\
\hline $\begin{array}{c}\text { Correlación por } \\
\text { continuidad }\end{array}$ & 4,694 & 1 &, 030 \\
\hline $\begin{array}{c}\text { Razón de } \\
\text { versomilitud }\end{array}$ & 6,696 & 1 &, 010 \\
\hline N de casos válidos & 45 & & \\
\hline
\end{tabular}

Los resultados nos permiten establecer la existencia de diferencias estadísticamente significativas entre hombres y mujeres en relación a la percepción de actividad $\left(X^{2}=6,318^{a} ; p=\right.$ ,012) siendo los hombres los que se perciben, casi en su totalidad (92\%), como más activos.

Por último, pretendemos relacionar las variables optimismo-pesimismo y la percepción de actividad, intentando establecer las posibles diferencias de los perfiles disposicionales y percibirse o no como un ejercitante activo. Para ellos realizaremos nuevamente el análisis de Chi-cuadrado.

Tabla 5.

Tabla de contingencia entre los perfiles disposicionales y la percepción de actividad

\begin{tabular}{|l|c|c|c|}
\hline \multirow{2}{*}{ Variable } & \multicolumn{2}{|c|}{ Percepción de actividad } & \multirow{2}{*}{ Total } \\
\cline { 2 - 3 } & \multicolumn{2}{|c|}{ Activo Pasivo } & \multirow{2}{*}{} \\
\hline Pesimista & 7 & 1 & 8 \\
\hline Optimista & 27 & 10 & 37 \\
\hline & 34 & 11 & 45 \\
\hline
\end{tabular}

Los resultados nos permiten observar que hay un predominio de personas optimistas que se perciben como activas (27 de 45), mientras que hay 10 , que son optimistas pero pasivos. En relación al análisis de Chi-cuadrado, encontramos que no existen diferencias estadísticamente significativas entre el optimismo-pesimismo y la percepción de actividad $\left(\left(X^{2}=, 752^{\mathrm{a}} ; p=, 386\right)\right.$.

\section{DISCUSIÓN Y CONCLUSIONES}

El objetivo de este trabajo se centra en el estudio de los perfiles optimista-pesimista en ejercitantes de una plaza urbana comunitaria, así como la percepción de actividad que tienen de sí mismos. Los resultados muestran diferencias estadísticamente significativas entre el factor optimismo y pesimismo de nuestra muestra, siendo el factor optimista el que obtiene puntuaciones más elevadas y está presente en una amplia mayoría de los participantes. Esto podría hacer referencia a lo planteado por Sheier et al.,(1985) indicando que los optimistas son personas que tienen expectativas y percepciones positivas 
sobre su vida, teniendo un mayor control de situaciones estresantes, regulando y reduciendo sus efectos negativos (Scheier \& Carver, 2006). Un ejemplo de situación estresante podría ser la realización de ejercicio físico (ya sea recreativo y/o competitivo) existiendo diferencias en el uso de estrategias de afrontamiento para lidiar con dicho estrés entre optimistas y pesimistas.

Así mismo, la percepción de control, podría ser entendida como una evaluación objetiva de la actividad física que realiza cada ejercitante (Ortín et al, 2011), siendo los hombres los que presentan una mayor percepción de actividad en relación a las mujeres, hecho que no se corroboró con la ejecución real de actividad, sino meramente con la percepción de la conducta de cada participante.

En cuanto a la relación percepción de actividad y el perfil disposicional, sea optimista o pesimista, los resultados nos permiten observar que hay un predominio de personas optimistas que se perciben como activas. El optimismo disposicional, alude a estrategias de afrontamiento positivas (planificación, reinterpretación positiva y crecimiento personal, afrontamiento focalizado al problema), es decir a conductas más adaptativas, (Murphy \& Tammen, 1998). No obstante, no se encontraron diferencias estadísticamente significativas entre el perfil disposicional y la percepción de actividad, por lo que aún no podremos corroborar la hipótesis de que el optimismo podría generar una mayor percepción de actividad. En futuras investigaciones, se podría proponer, la comparación de una muestra de personas activas y no activas físicamente, para poder así, obtener una mayor explicación de los resultados encontrados.

La importancia de este trabajo radica, en evidenciar la necesidad de generar hábitos de conducta activos en relación a la salud y calidad de vida en todas las etapas de la vida. Realizar actividad física de forma adecuada y saludable está relacionada con el aumento de la calidad de vida, incrementando en salud física, mental y social y disminuyendo factores de riesgo como el sedentarismo y sus consecuencias negativas para la salud integral de las personas (Weinberg y Gould, 2011). Lamentablemente, realizar actividad física regularmente es uno de los hábitos menos instaurados en la población en general. Por esta razón, los que realizan dicha actividad son un grupo minoritario, que seguramente cuenten con la fortaleza de la perseverancia muy desarrollada, aspecto que los podría ayudar a ser optimistas y en general a auto-percibirse como individuos activos.

En relación a las limitaciones encontradas en este estudio, nos encontramos primeramente con una muestra reducida, pero que de alguna manera expresa las características del lugar donde se ubica la única plaza urbana de Montevideo. El tamaño de la muestra no nos permite realizar determinados análisis estadísticos que nos posibilitarían a adaptar y baremar el instrumento utilizado a nuestra cultura, resultando esto una limitación importante, pues estamos analizando el perfil optimista-pesimista con un instrumento que no creado para nuestro medio. Por último hacer referencia a la falta de cuestionarios para valorar la percepción de actividad, reduciendo su uso, únicamente a posibles preguntas que puedan resultar de interés y/o de aproximación a esta temática.

Para finalizar, consideramos que al analizar el perfil de usuarios de los parques, se podrían implementar más actividades que respondan tanto al perfil optimista, de manera que éstas se adapten a su estilo y preferencias, así cómo incluir otras actividades, que puedan resultar de interés y que hasta ahora no han sido contempladas por personas con perfil pesimista.

Asimismo, sería muy interesante estudiar a la población en general en sus perfiles optimistas y pesimistas, ya que pareciera que las personas que realizan actividades físicas tienen altos grados de optimismo. De este modo, pensando en una relación recíproca entre las variables optimismo-actividad física, se podría promover en la población en general el desarrollo de la actividad física como medio para mejorar sus niveles de optimismo, o trabajar en mejorar su optimismo para poder iniciar conductas de autoeficacia y autocuidado como lo sería la actividad física.

\section{REFERENCIAS}

Abramson L. Y., Seligman M. E. P. y Teasdale J.(1978). Learned helplessness in humans: Critique and reformulation, Journal of Abnormal Psychology, 87, 32-48.

Bandura, A. (1982). Self - efficacy mechanism in human agency. American Psychologist, 37 (2), 122-147.

Bárez, M. (2002). Relación entre la percepción de control y 
adaptación a la enfermedad en pacientes con cáncer de mama. Tesis Doctoral no publicada. Universitat Autónoma de Barcelona. Bellaterra: Barcelona.

Beck, A. T. (1967). Depression: Clinical experimental and theoretical aspects. New York: Harper \& Row

Carver, C., Harris, S., Lehman, J. M., Durel, L. A., Antoni, M. N., Spencer, S. M. y Pozo, C. (2000). How important is the perception of personal control? Studies of early stage breast cancer patient. Personality and Social Psychology Bulletin, 26, 139-149.

Caspersen, C.; Powell, K. \& Christenson, G. (1985). Physical Activity, Exercise, and Physical Fitness: Definitions and Distinctions for Health-Related Research. Public Health Report Mar-Apr, 100 (2), 126-131.

Chico, Librán, E. (2002). Optimismo como predictor de estrategias de afrontamiento. Revista Psicothema, $V$. 14 (3), pp. 544-550.

Fernández - Castro, J., Álvarez, M., Blasco, T., Doval, E y Sanz, A. (1998). Validación de la Escala de Competencia Personal de Wallston: Implicaciones en el estudio del estrés. Ansiedad y estrés, 4, 31-41.

Ferrando, P.J., Chico, E., y Tous, J.M. (2002). Propiedades psicométricas del test de optimismo Life Orientation Test. Psicothema, 14 (3), 673-680.

Isaacowitz, D.M. (2005). Correlates of well-being in adulthood and old age: A tale of two optimisms, Journal of Research in Personality 39, 224-244.

Martin-Krumm, C. P., Sarrazin, P. G., Peterson, C. y Famose, J. P. (2003). Explanatory style and resilence after sport failure. Personality and Individual Differences, 35, 1685- 1695.

Myers, L.B. y Steed, L. (1999). The relationship between dispositional optimism, dispositional pessimism, repressive coping and trait anxiety. Personality and Individual Differences, 27(6), 1.261-1.272.

Mroczek, D. K., Spiro III, A., Aldwin, C. M., Ozer, D. J. y Bosse, R. (1993). Construct validation of optimism and pessimism in older men: Findings from the normative aging study. Health Psychology, 12, 406-409.

Murphy, S. \& Tammen, V. (1998). In search of psychological skills. En J.L. Duda (ed.) (1998). Advances in sport and exercise psychology measurement. (pp 195-209). Morgantown, WV: Fitness Information Technology.

Ntoumanis, N. (2001). A step-by-step Guide to SPSS for Sport and Exercise Studies. Londres: Routledge.

Ortín, F.J., Garcés de los Fayos, E.J., Gosálvez, J., Ortega, E. y Olmedilla, A. (2011). Optimismo y ejecución en el deporte en situaciones adversas. Replicando a Seligman 1990. Revista de Psicología del Deporte, 20 (2), 1-11.
Ortín, F.J., Ortega, E., López, E. y Olmedilla, A. (2011). Estilos explicativos de los entrenadores de fútbol profesional en el análisis de la competición deportiva. Anales de Psicología (artículo aceptado no publicado).

Otero, J. M., Luengo, A., Romero, F., Gómez, J. A. y Catro, C. (1998). Psicología de la personalidad. Manual de prácticas. Barcelona: Ariel Practicum.

Romero Carrasco, A.; García-Mas, A. \& Robert J. (2009). Estado del arte, y perspectiva actual del concepto de bienestar psicológico en Psicología del Deporte. Revista Latinoamericana de Psicología, 41(2), 335-347.

Scheier, M. \& Carver, C. (2006) Dispositional Optimism and Physical Well-Being: The Influence of Generalized Outcome Expectancies on Health. Journal of Personality, 55 (2), 169-210, DOI: 10.1111/j.1467-6494.1987. tb00434.x

Scheier, M. F., Carver, C. S. y Bridges, M. W. (1994). Distinguising optimism from neuroticism (and trait anxiety, self mastery and self esteem): A reevaluation of the Life Orientation Test. Journal of Personality and Social Psychology, 67, 1.063-1.078.

Scheier, M. y Carver, C.S. (1985). Optimism, Coping, and Health: Assessment and Implication of Generalized Outcome Expectancies. Health Psychology, 4, 219-247.

Seligman, M. E. P. (2004). Aprenda optimismo. Madrid: Debolsillo.

Seligman, M. E. P., Nolen-Hoeksema, S., Thornton, N., y Thornton, K. M. (1990). Explanatory style as a mechanism of disappointing athletic performance. Psychological Science, 1, 143-146.

Seligman, M.E. \& Csikszentmihalyi, M. (2000). Positive Psychology: An introduction. American Psychologist, 55, 5-14.

Shapcott, J.B., Bloom, G.A., Johnston, K.M., Loughead, T.M. y Delaney, J.S. (2007). The effects of explanatory style on concussion outcomes in sport. NeuroRehabilitation, 22. 161-167.

Weinberg, R. \& Gould, D. (2011). Foundations of sport and exercise psychology. United States of America: Human Kinetics. Url: www.humankinetics.com

Weiner, B., Frieze, I. H., Kukla, A., Reed, L., Rest, S. y Rosenbaum, R. M. (1971). Perveiving the causes of success and failure. Morristown, NJ: General Learning Press.

Tutte Vallarino, V. \& Del Campo Vega, C. (2011). Optimismo y percepción de la actividad física en usuario de una plaza urbana de Montevideo. Ciencias Psicológicas V (2): 151-157. 